\title{
La politique étrangère de l'Union Européenne à l'épreuve des normes américaines
}

The European Union Foreign Policy and the Challenge of American Norms

Frédéric Charillon

\section{OpenEdition}

12 Journals

Édition électronique

URL : http://journals.openedition.org/conflits/740

DOI : $10.4000 /$ conflits.740

ISSN : $1777-5345$

Éditeur :

CCLS - Centre d'études sur les conflits lilberté et sécurité, L'Harmattan

Édition imprimée

Date de publication : 1 décembre 2001

Pagination : 135-152

ISBN : 2-7475-2227-X

ISSN : 1157-996X

Référence électronique

Frédéric Charillon, «La politique étrangère de l'Union Européenne à l'épreuve des normes américaines », Cultures \& Conflits [En ligne], 44 | hiver 2001, mis en ligne le 22 mars 2006, consulté le 30 mars 2021. URL : http://journals.openedition.org/conflits/740 ; DOI : https://doi.org/10.4000/ conflits. 740

Ce document a été généré automatiquement le 30 mars 2021.

Creative Commons License 


\title{
La politique étrangère de l'Union Européenne à l'épreuve des normes américaines
}

\author{
The European Union Foreign Policy and the Challenge of American Norms
}

\author{
Frédéric Charillon
}

L'Union Européenne peut-elle résister à l'énorme rouleau compresseur américain qui s'est mis en marche, en matière de définition et de gestion de la sécurité et de la menace, au lendemain des attentats du 11 septembre 2001 ? La question peut paraitre indécente, alors que le Vieux Continent manifeste surtout sa sympathie et son soutien sincère à une Amérique touchée au cœur. C'est pourtant celle qui hante les couloirs bruxellois du Conseil européen ou de la Commission. C'est également celle qui occupe les esprits des décideurs au sein des diplomaties nationales.

Le problème de fond n'est pas nouveau. Depuis la fin de la guerre froide, les manifestations d'un certain unilatéralisme américain ne laissent pas d'inquiéter plusieurs pays Européens. Au temps de l'administration Clinton, les déclarations de Madeleine Albright sur «la nation indispensable ", sa gestion du dossier irakien par exemple, avaient donné le ton. Pendant la guerre du Kosovo en 1999, plusieurs désaccords transatlantiques étaient apparus, et la crise avait souligné les limites d'une Union Européenne qui n'existait qu'à travers les diplomaties nationales qui la composent (par exemple dans le groupe de contact). Ses moyens matériels étaient apparus limités, les «différences » qu'elle exprimait n'étaient aucunement prises en compte ${ }^{1}$. Le Conseil de Sécurité des Nations Unies n'ayant pas explicitement autorisé l'action militaire contre la Serbie, plusieurs diplomates européens avaient manifesté leur inquiétude face à un double glissement de la rhétorique américaine vers un registre politique (au nom de la sécurité) puis vers un registre moral (au nom des droits de l'Homme), dont les contours changeaient au gré des intérêts de Washington ${ }^{2}$. L'invocation un peu rapide d'une "communauté internationale » rassemblée derrière les Etats-Unis ${ }^{3}$, la banalisation de l'automaticité de la "solidarité atlantique » convoquée à l'initiative de la seule Amérique, avaient suscité des réactions de méfiance 
sinon de défiance. Et le ministre français des Affaires étrangères, Hubert Védrine avait exprimé sa préoccupation que ce conflit kosovar ne constitue pas un précédent mais bien plutôt une exception, dans l'approche des conflits à venir" ${ }^{4}$. Vint enfin l'élection de George W. Bush en novembre 2000, son style, l'orientation «dure» de son administration, et sa gestion unilatérale de dossiers comme ceux du protocole de Kyoto ou de l'initiative antimissiles.

L'événement du 11 septembre 2001 n'a fait que renforcer ces traits. La solidarité avec les Etats-Unis, plus que jamais, prend un caractère obligatoire. L'article 5 de l'OTAN a été invoqué. La souscription aux objectifs de guerre américains est quasi incontournable. La définition - sans cesse mouvante - de la menace telle qu'elle est formulée à Washington, est difficile à contester pour une diplomatie européenne ${ }^{5}$. Quelle est la signification, quel est l'impact de cette situation pour l'Union Européenne, et plus particulièrement pour la définition d'une politique étrangère et de sécurité commune crédible?

Il convient de rappeler trois points ici. D'une part, le choc international produit par les attentats du 11 septembre est intervenu au pire moment diplomatique pour l'Union Européenne. D'autre part, et en conséquence, l'Europe doit maintenant gérer un risque politique qui hypothèque l'avenir de sa diplomatie commune. Enfin, et néanmoins, la nouvelle situation internationale comporte un certain nombre d'opportunités, que les Européens auraient tort de ne pas saisir, à condition d'en avoir la volonté politique.

Un choc exogène dans un moment difficile

Les répercussions de ces attentats sur le système international, l'entreprise américaine de coalition building puis de norms buildingqui est mise en œuvre depuis le mois de septembre, les reclassements diplomatiques, sont autant d'éléments nouveaux appelant des réponses en matière de politique étrangère. La PESC était-elle prête?

La politique étrangère et de sécurité commune telle qu'elle a été définie au titre $\mathrm{V} d u$ traité de Maastricht, amendée au fil des traités ultérieurs (Amsterdam, Nice) ou forgée par les différentes avancées politiques (notamment le sommet franco-britannique de Saint-Malo en décembre 1998) ${ }^{6}$, reste un objet mal défini. Plutôt que de remplacer ou guider les politiques étrangères nationales, elle se superpose à elles dans un ordre de priorité et avec un degré de contrainte qui peut varier selon les thèmes abordés et les régions du monde concernées. On connaît, en outre, les principales difficultés qui entravent encore son fonctionnement : a) un processus décisionnel encore obscur, avec des rivalités de compétences qui ouvrent la voie à de multiples confusions, aussi bien pour les Européens eux-mêmes que pour leurs interlocuteurs extérieurs; b) une cohérence qui reste à trouver entre les différentes dimensions de l'action extérieure (diplomatie pure, commerce extérieur, aide au développement ou à la reconstruction, élargissement, dimensions militaires...) ; c) un fossé persistant entre d'une part les attentes que peut légitimement faire naître l'immense capacité financière de l'Union et d'autre part la faiblesse de son engagement ou tout du moins de son autorité politique, dans les dossiers importants qui ont marqué les relations internationales des années 1990 (de la Bosnie au Kosovo ou à la Macédoine, en passant par l'Afrique des Grands Lacs, la Tchétchénie ou le Proche Orient) ${ }^{7}$, d) enfin une volonté politique qui fait parfois défaut, tant il est difficile de rassembler tous les Etats membres autour des mêmes objectifs ou des mêmes intérêts, à partir de priorités souvent divergentes.

En dépit des priorités annoncées lors des Conseils européens d'Amsterdam puis de Nice, ces points n'avaient aucunement été réglés. Il était donc probable que la réponse 
européenne aux attentats du 11 septembre laisse apparaître les mêmes faiblesses et les mêmes contradictions. Et l'on a vu, en effet, l'Union Européenne en tant que telle s'effacer derrière les initiatives nationales. Dix ans après la guerre du Golfe de 1990-91, l'Europe en est-elle donc toujours au même point?

A la décharge de l'Union, le contexte était une nouvelle fois défavorable, pour au moins deux raisons. D'une part, l'exigence d'une réponse diplomatique aux attentats du 11 septembre intervenait à un moment où la PESC, après Nice et dans la foulée de la crise du Kosovo, entamait un second souffle (avec notamment la mise au point du concept de PESD - politique européenne de sécurité et de défense) ${ }^{8}$, sans pour autant être encore établie dans ses nouvelles marques. L'outil de défense européen n'est pas prêt, et les nouveaux organes politiques et militaires pour la conduite de la politique étrangère et de défense commune (le Comité politique et de sécurité 9 , le Comité militaire ${ }^{10}$ et l'étatmajor européen ${ }^{11}$ ), prévus en 1999, mis en place à titre provisoire en mars 2001 et devenus permanents au Conseil de Nice, n'étaient pas encore pleinement effectifs et encore moins rodés. D'autre part, la nomination récente de Javier Solana (octobre 1999) comme Secrétaire général du Conseil et Haut représentant pour la PESC, n'a pas complètement résolu les rivalités de compétence croissantes entre les différentes personnes habilitées à parler au nom de l'Union. Ainsi, en réaction aux attentats, on a $\mathrm{pu}$ enregistrer une grande effervescence de la part de plusieurs acteurs, qui ont proposé autant d'analyses personnelles de la situation. Le Secrétaire Général du Conseil Javier Solana, tout en multipliant les voyages (notamment au Moyen-Orient - Israël, territoires palestiniens, Egypte, Jordanie, Arabie Saoudite, les 22-25 octobre 2001), s'est largement exprimé. Dans un discours tenu à Rimini, le 21 octobre, il a tenu des propos ambigus sur le rôle de la culture dans les conflits actuels ${ }^{12}$, insisté sur la faillite de l'Etat dans le développement de ces conflits ${ }^{13}$, et insisté sur la dimension purement afghane de la crise actuelle (plutôt, par exemple, que sur la dimension moyen-orientale) ${ }^{14}$. D'autres ont exploré des pistes différentes. Ainsi le président de la Commission, Romano Prodi, qui n'a pas pour autant été en mesure de tirer avantage du conflit pour faire oublier le discrédit dans lequel il est tenu par un nombre croissant de chefs d'Etat et de gouvernement de l'Union. Le commissaire britannique chargé des relations extérieures, Chris Patten, a continué, avec un certain franc-parler, de proposer ses propres analyses ${ }^{15}$. Enfin, la présidence belge de l'Union (en fonction jusqu'à fin décembre 2001) ne s'est pas privée de profiter de l'occasion pour faire entendre «sa " diplomatie ${ }^{16}$.

Cela ne signifie pas pour autant que la diplomatie de l'Union Européenne ait été totalement absente de la crise. L'Europe, en tant que telle, fut même beaucoup moins absente qu'elle ne le fut dans la guerre du Golfe voici dix ans. Ce qui laisse penser que la création de la PESC à Maastricht puis ses développements ultérieurs n'ont pas été vains. Sur le fond, et en dépit d'une certaine cacophonie, que peut-on retenir? Il faut, pour répondre à cette question, tenir compte des positions du Conseil, organisme véritablement habilité à formuler des positions en matière de PESC, plutôt que de celles de la Commission ou du Parlement Européen, sauf naturellement lorsque celles-ci, par leur décalage avec les positions du Conseil, sont de nature à brouiller à l'extérieur la perception d'une identité européenne en matière de diplomatie. Plusieurs éléments se dégagent alors de ce que l'on peut, tant bien que mal, qualifier de « discours européen sur l'après 11 septembre ».

1- Le soutien aux Etats-Unis a été largement confirmé, dès le 11 septembre. Le 14 septembre a été décrété jour de deuil en Europe, puis, le 20 septembre, l'Union et les 
Etats-Unis, dans une déclaration conjointe, se sont engagés sur une série d'actions communes pour lutter contre le terrorisme ("Cette agression contre l'humanité a frappé au coeur d'une nation amie avec qui l'Union européenne partage le combat pour un monde meilleur »). Un Conseil européen extraordinaire a reconnu le 21 septembre la légitimité d'une riposte américaine aux attentats, sur la base de la résolution $1368 \mathrm{du}$ conseil de sécurité des Nations Unies. Le Conseil européen a également appelé à la formation d'une coalition internationale aussi large que possible sous l'égide de l'ONU pour lutter contre le terrorisme

2- Pour autant, la résistance à l'unilatéralisme a été répétée. Les Européens ont insisté sur le rôle central de l'ONU, plutôt que sur l'extension possible des missions de l'OTAN. Des inquiétudes ont été exprimées également sur la conduite de la guerre par les EtatsUnis, notamment sur l'utilisation de bombes à fragmentation.

3- Il est enfin important de souligner que l'Union européenne rejette clairement et explicitement tout amalgame entre terrorisme et monde arabe et/ou islamique, ainsi

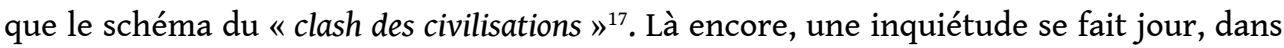
les cercles diplomatiques européens, sur la possibilité d'une dérive en ce sens si certains courants de l'administration américaine, marqués à la fois par l'unilatéralisme et par une vision plus manichéenne du monde, prenaient l'ascendant. On craint ainsi, pour être plus précis, des frappes américaines sur d'autres pays, surtout sur l'Irak si ce dernier pays était tenu responsable des derniers événements par Washington. De telles frappes auraient plusieurs répercussions négatives : elles feraient entrer les Etats-Unis dans le jeu d'un affrontement "Occident contre monde musulman »; elles susciteraient une radicalisation de la rue arabe, ce qui mettrait plusieurs régimes du Sud et de l'Est de la Méditerranée, partenaires de l'Union Européenne dans le cadre du processus de Barcelone, dans une posture plus que délicate; enfin elles provoqueraient sans aucun doute des dissensions au sein même de l'Union, notamment entre les plus atlantistes (Grande-Bretagne, Pays Bas, Portugal...) et les autres.

La politique étrangère européenne dans la tourmente

L'Europe a-t-elle les moyens de s'opposer à des orientations imposées par la diplomatie américaine, et qui seraient néfastes pour ses intérêts ? A-t-elle les moyens de proposer des alternatives à de telles orientations, et de trouver des partenaires internationaux pour la suivre dans ses éventuelles initiatives? Si l'une des définitions de la puissance est d'avoir la capacité "de faire, de faire faire, et d'empêcher de faire», force est de constater que l'Union Européenne se trouve bien démunie en la matière... L'Europe courait plusieurs risques dans cette affaire. Aucun d'eux n'a véritablement été évité.

Le premier risque, pour les Européens, était celui de se comporter comme une caricature d'eux mêmes, c'est-à-dire de se contenter de vagues formules insignifiantes qui ne reflètent jamais que la difficulté éprouvée par les partenaires à s'entendre sur autre chose. Et l'on retrouve bien, dans les réactions aux attentats, les déclarations creuses et faussement consensuelles, typiques de la PESC et qui provoquent traditionnellement la fureur de ses détracteurs parce qu'elles n'expriment aucune volonté politique véritable. Ainsi l'Union:

"reconnait la nécessité de développer un dialogue approfondi avec les pays et régions du monde où le terrorisme prend racine"; elle" souligne également qu'il faut intégrer tous les pays dans un système mondial équitable de sécurité, de prospérité et de meilleur développement, permettant[...]l'émergence d'une communauté internationale forte et soudée pour lutter contre le terrorisme ${ }^{18}$. 
Le second risque résidait dans l'éventualité d'une rivalité entre les diplomaties nationales. On sait, depuis la guerre du Golfe, l'importance que peut prendre le timing d'une crise internationale au regard des rapports de force interne à l'Europe, entre les diplomaties et les défenses nationales ${ }^{19}$. Apparaître comme un acteur clef de la future défense européenne (en termes de moyens) ou au contraire comme un maillon faible, n'est pas sans conséquences pour les discussions à venir sur l'organisation de la défense européenne. Or, tout est effectivement mis en œuvre à Paris, Londres ou Berlin, pour mettre en avant son savoir faire, ou à l'inverse masquer ses carences. Les trois principales diplomaties européennes ont été sans surprise parmi les plus promptes à réagir aux attentats et à se positionner comme les fers de lance de l'analyse européenne, mais on compte au total, sur les deux premiers mois de la crise (entre le 11 septembre et le 5 novembre), pas moins de 48 tournées ou missions effectuées séparément par les ministres des Affaires Etrangères européens au sujet de la crise afghane. Chacun n'envisage que son image nationale par rapport aux voisins, sans chercher à construire une option européenne.

A l'heure où l'unique porte-avion français Charles de Gaulle n'était pas opérationnel, et où l'armée française, dans sa transition vers une armée de métier, traverse une passe difficile, c'est avec une certaine crainte que Paris a abordé une crise dans laquelle Berlin se disait prête à envoyer 3900 hommes pour des opérations militaires en Afghanistan. La France a donc voulu présenter le 1er octobre «son " plan d'action, envoyé à tous les partenaires européens, présenté en Iran, en Inde et au Pakistan (par l'envoyé spécial et ancien ambassadeur Pierre Lafrance), et articulé essentiellement autour de quatre points : a) le renforcement de l'assistance humanitaire d'urgence, b) la nécessité de préparer une solution politique proprement afghane pour l'après Talibans, c) la préparation d'un plan de reconstruction à long terme et d) la préparation d'un plan de développement ${ }^{20}$. La Grande-Bretagne, de son côté, a mis en avant ses capacités militaires et sa solidarité infaillible avec Washington, vantées par un discours belliqueux. Cette attitude avait déjà été celle du Premier ministre Tony Blair dans la crise du Kosovo, ou dans la poursuite des frappes sur l'Irak. Il s'agit là de montrer la supériorité britannique en matière de capacité de réaction militaire, supériorité réelle à l'échelle européenne mais servie, en l'occurrence, par la tenue dans les jours précédents l'attentat d'un exercice militaire avec les forces du sultanat d'Oman dans l'Océan indien, qui était prévu de longue date et qui a réuni 24000 soldats britanniques : autant le timingde la crise était mauvais pour l'image militaire de Paris, autant il était bon pour celle de Londres.

Ces «petits meurtres entre amis » n'auraient pas autant d'importance s'ils n'avaient pour conséquence la division, le manque de volonté commune et la reprise de la course au leadership en Europe, cette dernière étant arbitrée savamment par les Etats-Unis. Les discours de Tony Blair ont ainsi valu à la Grande-Bretagne d'être qualifiée par George Bush d'ami «le plus sûr des Etats-Unis », ce qui est important pour Londres dans le contexte européen (on se souvient des craintes britanniques, après la réunification allemande, lorsque Bonn était en passe de devenir la priorité de politique extérieure de George Bush senior). Ses voyages nombreux, et ses initiatives diplomatiques (Asie Centrale, Inde-Pakistan début octobre, Moyen Orient du 31 octobre au 2 novembre, par exemple), ont visé à le placer au-dessus de ses homologues européen $\mathbf{s}^{21}$. Plus encore, cet activisme a agacé ses partenaires, en prenant les allures d'une gesticulation, d'une tentative pour présenter la Grande-Bretagne comme cobelligérante auprès de Washington alors qu'elle n'en a pas les moyens militaires, et 
d'un effort pour faire oublier que Londres demeure le sanctuaire d'un bon nombre d'associations islamiques désormais montrées du doigt par les autorités américaines. La Belgique ou les Pays Bas ont ainsi déploré les initiatives de Tony Blair, parfois avec force.

Cette situation empêche les Européens de définir une position unique. Les désaccords sont apparents, et les rivalités patentes. Mais un autre point important a desservi l'ambition d'une politique étrangère et de sécurité commune européenne, point qui sur le moment n'a sans doute pas été suffisamment analysé : il s'agit des tentatives piteuses effectuées pour créer une sorte de directoire des trois grandes diplomaties - France, Grande-Bretagne, Allemagne - autour de la gestion de cette crise. Défendue par certains diplomates depuis longtemps, l'idée qu'un triangle diplomatique européen francogermano-britannique était nécessaire s'était peu à peu imposée dans les esprits depuis le sommet franco-britannique de Saint-Malo en décembre $1998^{22}$. Ce triangle n'était pas vu comme un club fermé ayant vocation à reléguer au second rang les autres politiques étrangères, mais plutôt comme le moteur et la condition sine qua non d'une politique étrangère européenne crédible. Or, après le 11 septembre, les réunions à trois, présentées comme des sommets informels de l'Union, ont tourné au fiasco. Celle qui a eu lieu à Gand le 19 octobre suscita la réaction outragée de Romano Prodi, l'agacement des petits pays, la colère de l'Italie et de l'Espagne. Le 4 novembre, la nouvelle réunion proposée par Tony Blair à Londres, s'est terminée on ne peut plus mal pour l'image de l'Europe: le Premier ministre britannique fut obligé in extremisd'inviter Sylvio Berlusconi, puis José Maria Aznar, Guy Verhofstadt, Javier Solana, et le néerlandais Wim Kok, qui se sont imposés "à table " dans une atmosphère de règlements de compte publics. Pour finir, le président de la Commission Romano Prodi s'est réjouit ouvertement de la «mort du directoire à trois». Il oublie que cet échec est lourd de risques pour l'avenir de la PESC. Les « petits » pays reprochent désormais aux grands ce qu'ils considèrent comme une tentative arrogante pour confisquer la politique étrangère européenne ${ }^{23}$. Cela mine la légitimité d'un triangle Londres-Paris-Berlin en matière de politique étrangère, triangle que chacun s'accorde à considérer fondamental pour la mise au point d'une PESC digne de ce nom.

Le 11 septembre aura encore eu d'autres conséquences néfastes pour l'Union Européenne : son statut de pôle potentiel a pratiquement disparu, sa voix diplomatique est mise en veilleuse, et ses carences militaires sont mises en lumière. Les attaques contre le World Trade Center ont d'abord renforcé de manière spectaculaire le statut des Etats-Unis comme puissance structurante, par rapport aux ordres de laquelle s'articulent les réactions des décideurs et plus largement des élites européennes. Avec des débats nationaux articulés autour de thèmes tels que "nous sommes tous américains $»^{24}$ ou "nous devons solidarité à nos alliés", la question d'une réponse purement européenne ne se pose pas. La grille de lecture proposée par George Bush s'impose à tous : "si vous n'êtes pas avec nous, vous êtes contre nous ». Et avec la formule chère à Hubert Védrine " amis, alliés, mais pas alignés ", Paris apparait bien seul face aux Etats-Unis dans cette posture. Par ailleurs, en rivalisant de propositions séparéespour mettre sur le terrain des moyens militaires nationaux très limités, au service des EtatsUnis qui ne leur ont pratiquement rien demandé, les pays européens ne font que souligner les lacunes de leurs dispositifs un an à peine après la conférence sur les capacités militaires de l'Union, tenue à Bruxelles les 20 et 21 novembre 2000. Tandis que l'on discutait, dans les mois précédents, des modalités de la mise à disposition de moyens de l'OTAN pour d'éventuelles opérations militaires européennes communes à 
venir $^{25}$, la crise afghane inverse le processus: ce sont des forces européennes, nationales et séparées, qui viennent en faible appoint à la demande des Etats-Unis, lesquels gardent la maîtrise de l'agenda et des choix stratégiques.

Dans la guerre du Kosovo, la France s'était retrouvée seule dans l'Alliance atlantique pour demander que l'OTAN n'agisse pas comme gendarme du monde, en lieu et place des Nations Unies. Désormais, l'alternative est encore plus réduite : un débat est en train de naître aux Etats-Unis sur la question de savoir si l'OTAN est même encore utile à l'Amérique, ou si elle n'est plus qu'une structure encombrante, qui permet aux « petits » alliés européens d'avoir un mot - de trop - à dire. A la fin des années 1990, les Européens mettaient un point d'honneur à ciseler leur propre formulation d'une politique moyen-orientale différente de celle des Etats-Unis, leur propre définition de la criminalité internationale, leur propre agenda politique, leur propre approche de ce que les Etats-Unis appelaient les Rogue States, et avec lesquels l'Europe préférait le "dialogue critique " à la mise en quarantaine. Si contestables ou insuffisantes que soient certaines de ces tentatives, elles avaient le mérite d'exister. Plusieurs mois après le 11 septembre, l'agenda politique international américain et sa capacité à s'imposer semble laisser moins de marge de manœuvre que jamais à l'Union Européenne. Les "Etats terroristes ", ou "ennemis de la liberté », sont désormais définis comme ceux qui ne se plient pas à la rhétorique dominante. La double question de l'imposition des normes et de la capacité à imposer son discours sur la scène mondiale se pose avec une force peut-être sans précédent.

Les opportunités du 11 septembre : une marge de manœuvre possible pour l'Europe?

Les positions communes exprimées au nom des 15 au début de la crise sont restées floues, et insistaient surtout sur ce que l'Union savait faire le mieux : le financement de programmes de reconstruction. Le directoire à trois promu par Londres avec Paris et Berlin ayant tourné court, reste un axe franco-britannique qui s'efforce de conserver quelques positions communes ${ }^{26}$ mais sur fond de rivalité. Pourtant, des opportunités s'offrent aux Quinze, qui nous paraissent être de deux ordres :

1- L'opportunité d'une reprise de l'initiative européenne en direction de plusieurs blocs régionaux.

2- L'opportunité de ramener les derniers événements sur un terrain plus propice au savoir-faire de l'Union Européenne tel qu'on a déjà pu le mesurer dans les dernières années.

Les événements du 11 septembre peuvent d'abord permettre à l'Union Européenne soit de développer sa présence et sa visibilité dans des régions du monde dans lesquelles elle n'a jamais véritablement été influente, soit de renouer des liens qui s'étaient récemment relâchés. Avec l'Asie centrale et l'Asie du Sud, régions dans lesquelles l'Union n'a jamais eu de relation prioritaire, c'est essentiellement en termes d'assistance - et donc de présence - financière que la question est posée aujourd'hui. Le lancement de programmes de financement, de reconstruction ou d'encadrement, permet d'envisager de nouveaux horizons pour la diplomatie européenne, dans une zone qui depuis la fin de la guerre froide était devenue une sphère d'influence américaine. Avec la Russie, l'affaire est plus complexe. L'agenda américain de lutte contre le terrorisme arrange le président Poutine, élu grâce à une guerre en Tchétchénie qu'il a - au minimum - exploitée, mais qu'il ne maîtrise plus aujourd'hui, et qui lui avait valu une brouille diplomatique avec plusieurs pays européens dont la France. Le 11 septembre remet les compteurs à zéro. Le 3 octobre, l'Union européenne 
et la Russie se sont engagées à renforcer leur coopération politique et la lutte conjointe contre le terrorisme, sans vraiment définir ce dernier concept. Il ne fait aucun doute que la situation permet à la Russie de réduire les opposants tchétchènes à ce vocable de "terroristes ", et ainsi masquer à la fois la brutalité militaire et l'impasse politique, ce que ne peut soutenir l'Union Européenne. Mais dans le même temps, c'est précisément ce flou de la notion de terrorisme qui permet aux deux parties de tirer avantage de la nouvelle donne pour relancer un dialogue euro-russe que l'on pressent nécessaire à l'heure où les Etats-Unis renforcent encore leur emprise sur l'agenda politique mondial. Le 20 octobre, avec la Russie, l'Union a réuni les 13 pays candidats à l'adhésion, les pays de l'Espace économique européen (Norvège, Islande, Liechtenstein), la Suisse ainsi que l'Ukraine, la Moldavie et les pays des Balkans occidentaux, dans le cadre d'une "Conférence européenne" pour coordonner leurs politiques de lutte contre le terrorisme international. Le prix à payer, pour les Quinze, est de «lâcher » la cause tchétchène pour la mettre dans la catégorie des "défis communs » et ainsi accepter de fait la rhétorique russe en la matière ${ }^{27}$. Le gain étant la reprise d'une dialogue qui était devenu difficile avec Moscou.

Une limite de taille, cependant, à ce premier type d'opportunité : le Moyen-Orient. Avec l'Europe centrale et orientale, cette partie du monde fait partie de "l'étranger proche " de l'Union Européenne ${ }^{28}$, pour reprendre une notion issue de l'analyse de la politique étrangère de l'ancienne Union Soviétique ${ }^{29}$. Elle aurait sans aucun doute souhaité saisir l'occasion d'y exercer une plus grande influence. Les premières semaines qui ont suivi les attentats ont laissé penser que tel serait le cas: les Européens multipliaient les missions dans la région, un Forum Méditerranéen s'est tenu à Agadir les 25-26 octobre, les déclarations se sont succédées ${ }^{30}$, et une rencontre Arafat-Peres a même pu se tenir sous l'égide de l'Union Européenne ${ }^{31}$. Plus encore, les Etats-Unis eux-mêmes semblaient preneurs de toute aide extérieure capable de les aider à tenir le front «antiterroriste ». L'Union, qui bénéficie d'une bonne image dans le Moyen-Orient arabe et qui reste le premier bailleur de fonds de l'Autorité Palestinienne depuis le début du processus d'Oslo, pouvait jouer ce rôle : le président Arafat a d'ailleurs une nouvelle fois demandé à l'Union Européenne d'être plus présente sur le dossier du Proche Orient. Mais la "fenêtre d'opportunité » s'est vite refermée. A peine la victoire militaire était-elle assurée en Afghanistan que l'administration américaine revenait à un unilatéralisme dur, revenant sur ses déclarations d'ouverture à l'égard du monde arabe en général et des Palestiniens en particulier, pour assurer un soutien inconditionnel et l'impunité totale au Premier ministre israélien Ariel Sharon, y compris dans sa politique d'assassinats « ciblés » de dirigeants politiques adverses, dans sa politique de punitions militaires collectives contre des civils, et dans son choix de rompre tout dialogue avec la partie adverse ${ }^{32}$. Que peut alors faire l'Europe, sinon dénoncer le jusqu'au-boutisme israélien ${ }^{33}$, et payer pour faire reconstruire les infrastructures civiles détruites volontairement par Tsahal?

Il reste cependant une autre carte aux Européens : celle qui consiste à ramener les événements sur le terrain le plus propice à leur savoir faire.

Car le contexte marqué par un discours de lutte multiforme et de longue haleine contre un ennemi difficile à cerner, qui nécessitera davantage de réflexion, de concertation et de programmes à long terme que d'action militaire pure, massive et ponctuelle, constitue un terrain favorable pour l'Union Européenne. La PESC, telle qu'elle a pu être 
observée dans les crises internationales successives des années 1990, comporte en effet quatre caractéristiques au moins. A savoir :

a) elle est une "politique étrangère de proximité ", c'est-à-dire une politique qui développe des actions visibles sur le terrain pour les populations concernées (monitoring d'élections, financement de routes, d'hôpitaux, de structures portuaires etc.), autant que des actions impliquant seulement les chancelleries ${ }^{34}$.

b) elle développe une "diplomatie du créneau ", c'est-à-dire qu'elle intervient d'abord en complémentarité avec les Etats-Unis, sur les dossiers délaissés par ces derniers, ou lorsque aucune "grande puissance» (Russie, Chine ou autre) ne revendique une "sphère d'influence ${ }^{35}$.

c) elle joue surtout un rôle de bailleur de fonds incontestable (notamment dans les Balkans et au Moyen-Orient).

d) elle est une politique étrangère proactive plutôt que réactive, qui sait inventer des cadres d'action préventifs ou structurants (comme le pacte de stabilité pour l'Europe orientale dans les années 1993-94, ou encore le lancement du partenariat euroméditerranéen), davantage qu'elle ne parvient à réagir rapidement et efficacement à un choc international exogène ${ }^{36}$.

La situation actuelle correspond plutôt bien à ce "savoir faire " de la PESC. Javier Solana ne s'y est d'ailleurs pas trompé (lors du Conseil informel des ministres de la défense le 12 octobre) :

"As the causes of conflict becomes more complex, so our approach to prevention has to be more inventive. And terrorist, unconventional warfare calls for unprecedented responses. As we have seen over the last few weeks, the military is only one element of that response. We in the EU have reacted to the terrorist attacks by undertaking an ambitious range of measures, many of them in the area of internal and judicial affairs, and of control of financial flows. We are in particular having to look again at how we use information, both open and covert, how we share it, and how we protect it. Preventing conflict depends more than ever on access to good intelligence. [...] We also have to look deeper into the causes of conflict. As it is increasingly born out of failed states, a key option for peace has to involve assisting such states to rebuild themselves. [...] The European Union is in a unique position to address this issue ».

Les premières réponses européennes imaginées pour la région Asie Centrale ont été formulées en termes d'assistance et d'accords de coopération et de renforcement de l'assistance déjà existante (par exemple pour le Pakistan). En insistant sur la nécessité de développer des réponses "pas uniquement militaires " (comme l'a encore rappelé Jacques Chirac le 5 novembre), en rappelant sans cesse la « complexité » de la riposte, son caractère " global ", l'Union s'efforce de faire passer le dossier sur un terrain qui lui est plus favorable que celui de l'opération militaire, pour laquelle elle n'est pas prête. On pourra dire que depuis le Kosovo, l'Europe n'a que peu innové : elle compte toujours sur ses mêmes points forts, qui sont sa capacité d'organiser un grand nombre de réunions, et sa capacité à financer des programmes d'action. Il n'en demeure pas moins que ces deux atouts peuvent être utiles pour gérer l'après crise.

La notion de terrorisme n'est pas définie, ni encore moins balisée juridiquement, mais elle s'impose aux ennemis des Etats-Unis. L'activation de l'article 5 de l'OTAN a été décrétée, et l'Union Européenne apporte un soutien sans réserve à Washington, tandis que les diplomaties nationales rivalisent de zèle pour participer à la "guerre contre la terreur ». Il est des matins radiophoniques où le malaise du président américain après 
l'ingestion d'un bretzel est plus important que les victimes civiles des conflits irakien, afghan et palestinien réunis. Vu du Vieux continent, le monde de l'après 11 septembre est bel et bien formulé par les Etats-Unis.

Mais la question qui se pose aujourd'hui à l'Europe n'est pas normative (« l'Europe a-telle raison de soutenir son allié américain?»). Les deux continents sont alliés, les attentats étaient inexcusables, et après tout, on peut en penser ce que l'on veut. La science politique n'a pas à en juger. La question est bien plutôt : « l'Europe a-t-elle les moyens de développer une autre attitude, ou bien se trouve-t-elle déjà dans l'obligation d'apporter un soutien inconditionnel à Washington, au point de fermer les yeux sur ses excès? ». C'est le débat sur la puissance, sur sa définition, et sur ses instruments, qui est à nouveau posé. Et en la matière, le monde de l'après 11 septembre s'annonce bien complexe. La hard power semble triompher, mais à condition d'avoir sur le terrain des alliés pour faire la guerre par procuration. ${ }^{37}$ L'imposition des normes est plus importante que jamais, et les Etats-Unis ont imposé les leurs. Mais c'est Al Jazira qui a informé le monde, plutôt que $\mathrm{CNN}$ : pour la première fois, et contrairement au Golfe ou au Kosovo, il y a véritablement deux espaces de sens face à face ${ }^{38}$, avec chacun ses moyens d'information. Entre hard power et "puissance civile », entre CNN et Al Jazira, l'Europe peut-elle se faire une place ? Ou sera-t-elle absorbée par son alliance avec les Etats-Unis, en faisant de plus en plus semblant de l'avoir choisie elle-même ( Puisque ces événements nous dépassent... ») ? Paradoxalement, c'est la marche de l'administration Bush vers la prophétie auto réalisatrice du Clash des civilisations - plus par ignorance et myopie domestique que par complot idéologique - qui offre un créneau à l'Union Européenne. Il y a aujourd'hui une demande d'Europe, du Caire à Ramallah. Il y a également une nécessité d'Europe pour le système international. Mais cela implique, de la part des Européens, une capacité d'initiative plus forte et plus réactive que celle qu'ils se sont forgée jusqu'à présent.

\section{NOTES}

1. . C'est Washington qui a mené la danse, sans considération pour les préférences du Vieux Continent, qui aurait aimé, notamment, un traitement plus neutre ou moins manichéen du conflit, et moins complaisant envers l'UCK.

2. . Voir Corten, O., Delcourt, B. (eds.), Droit, légitimation et politique extérieure : l'Europe et la guerre du Kosovo, Bruxelles, Bruylant, 2001.

3. . En fait de communauté internationale, ni la Russie, ni la Chine, ni le Brésil, ni l'Ukraine, ni l'Afrique du Sud, ni le groupe de Rio, ni bien d'autres Etats encore, n'ont signé les principales résolutions des Nations Unies sur le Kosovo, notamment la résolution 1244.

4. . Voir notamment l'entretien de Hubert Védrine avec la presse indienne, le 18 février 2000, consultable sur la base documentaire du quai d'Orsay : http:// www.diplomatie.gouv.fr

5. Ce constat n'est pas uniquement celui des alliés américains de Washington, ni encore moins - l'expression d'un anti-américanisme français. Il est également dressé 
par des observateurs américains, en termes d'ailleurs parfois plus durs encore. Voir les articles de William Pfaff dans le Herald Tribune, plus particulièrement : " The politics of terrorism, or civilians vs civilians », IHT, 10 janvier 2002 ; « Will the new world order rest solely on American might? », IHT, 29 décembre 2001 ; « In its quest for supremacy, U.S. may squander partnerships », IHT, 15 décembre 2001.

6. . Voir F. Charillon, « De Suez à Skopje : un nouveau partenariat britannique pour le XXIe siècle?", Politique Etrangère, hiver 2001-2002.

7. . L'expression « Expectation / Capabilities Gap » est de Christopher Hill, enseignant à la London School of Economics, et dont on pourra consulter l'ouvrage majeur en la matière : Ch. Hill (ed.), The actors in Europe's foreign policy, Londres, Routledge, 1996. 8. . Voir F. Charillon, "L'Union Européenne en 2000 : le retour des intérêts nationaux ", in A. Grosser (dir.), Les pays d'Europe occidentale, Paris, La Documentation Française, 2001.

9. . Composé de représentants permanents avec rang d'Ambassadeurs, ayant vocation à traiter, en temps normal, de l'ensemble des questions liées à la PESC. Dans le cas d'une opération militaire en temps de crise, il exercera sous l'autorité du Conseil, le contrôle politique et la direction stratégique de l'opération.

10. . Composé des Chefs d'état-major des armées, représentés par leurs délégués permanents. Il donnera des avis et formulera des recommandations destinées au COPS, et fournira des instructions militaires à l'Etat-major de l'UE.

11. . L'État-major intérimaire, comprenant initialement environ 20 experts militaires et 8 secrétaires civils, va s'étoffer pour atteindre à terme 45 membres. Il a été décidé en novembre 2000 de confier sa direction à un général allemand, Rainer Schuwirth, contre une candidature française, ce qui a suscité une nouvelle tension entre les deux pays. Il mettra ses compétences militaires au service de la PECSD et sera chargé de l'alerte rapide, de l'analyse de situations, et de la planification stratégique pour les missions dites de Petersberg.

12. . «Whether it be in Bosnia, Croatia, Timor, Chechnya or Rwanda, ethnic and religious dividing lines have been at the heart of conflict. Both ethnicity and religion are powerful symbols of identity, and provide a rallying point during times of uncertainty or external pressure. But they are frequently misused, and become themselves part of the problem. The result is that conflicts are now rarely the result of declarations of war between legitimate governments. Instead they arise from disparate groups of disaffected peoples who see no alternative method of addressing their perceived grievance, be it political, economic or religious ». " The fire and the crystal ». Conférence, Rimini, Italie, 21 octobre 2001, n S0166/01, disponible à l'adresse : http:// ue.eu.int/solana/details.asp ?BID=107\&DocID=68095

13. . « Conflict is increasingly born out of failed states. Failed both politically and economically ». Ibid.

14. . « [...] Finally, whether we like it or not, we are all increasingly caught up in conflicts, even when their origins are geographically distant. [...] The result is that international issues now almost always have a domestic element. [...] Almost in every European household, Afghanistan is today at the heart of our daily preoccupations ». Ibid.

15. . L'interview donnée par ce dernier sur Arab News Network, le 18 octobre (après avoir publié un article dans le Financial Time la veille), proposait ainsi une vision claire mais toute personnelle des événements : 1 - ce n'est pas une guerre entre l'islam et l'Occident, 2- il s'agit d'une campagne anti-terroriste de longue haleine, sur plusieurs 
fronts, qui doit être menée sous l'autorité des Nations Unies, 3- l'aspect militaire de cette campagne devra rester proportionné et ne pas engager de moyens extrêmes, 4parallèlement, l'Europe [selon Chris Patten] veut voir le plan Mitchell mis en œuvre au Proche Orient, impliquant notamment la création d'un Etat palestinien (établissant ainsi un lien avec cette région du monde et minimisant la dimension purement afghane, contrairement à Javier Solana).

16. . Le Premier ministre Verhofstadt a ainsi tenu le 23 octobre un discours à Varsovie sur « Le nouvel ordre mondial après le 11 septembre ». Le 1er novembre, il avait évoqué une possible pause dans les bombardements pendant le ramadan, allant ainsi à l'encontre de ce que répétaient les Etats-Unis et la Grande-Bretagne M. Michel s'était déjà illustré, dans les jours précédents, par les critiques qu'il avait formulées à l'encontre des « gesticulations » britanniques.

17. . Si l'on excepte, bien entendu, les déclarations toujours inconvenantes du président du Conseil Italien, Sylvio Berlusconi, sur la supériorité de la civilisation occidentale par rapport à la civilisation musulmane... Pour la version dure et non expurgée du « clash des civilisations ", voir S. Huntington, « The Clash of Civilizations? », Foreign Affairs, n 72(3), été 1993.

18. . Source : Conseil européen.

19. . La crise de 1990-91 avait démontré la plus grande marge de manœuvre de la France par rapport à l'Allemagne, quelques mois après la réunification, pour conduire des interventions extérieures. Mais elle avait aussi marqué les difficultés françaises à envoyer sur le terrain un nombre d'hommes conséquent (environ 15 000), alors que la Grande-Bretagne avait pu en déployer plus de 30000.

20. . L'activisme politique français s'est aussi reflété dans les multiples contacts téléphoniques du président Chirac, qui s'est rendu deux fois aux Etats-Unis ; le ministre des Affaires étrangères, Hubert Védrine, a également multiplié les déplacements, notamment en Inde, au Pakistan, dans le Golfe, au Maghreb, en Israël et dans les territoires palestiniens.

21. . Même si son voyage au Moyen-Orient, notamment en Syrie, a été particulièrement infructueux.

22. . F. Charillon, «L'Union Européenne en 1999 : sous la pression des événements », in A. Grosser (dir.), Les Pays d'Europe Occidentale, op. cit.

23. . Depuis la difficile repondération des voix au Conseil de Nice, le fossé entre « grands » et " petits » étaient déjà patent. Voir F. Charillon, « L'Union Européenne en 2000 : le retour des intérêts nationaux », in A. Grosser (dir.), Les pays d'Europe occidentale, op. cit. Ainsi que D. Galloway, The Treaty of Nice and Beyond. Realities and Illusions of Power in the EU, Sheffield Academic Press, Sheffield, 2001.

24. . Comme n'avait pas hésité à le proclamer Jean-Marie Colombani dans son éditorial du Monde au lendemain des attentats (copiant ainsi l'expression formulée sous le coup de l'émotion la veille au soir à la télévision par la chercheuse Nicole Bacharan).

25. . Dans la lignée des discussions qui avaient eu lieu sur les Combined Joint Task Forces en 1993, puis sur la coordination UE-OTAN depuis le sommet de l'OTAN à Berlin en 1996, jusqu'aux arrangement dits « Berlin Plus » qui élargissent sur le papier une coopération encore jamais testée sur le terrain.

26. . Par exemple, la déclaration de presse conjointe de Jacques Chirac et de Tony Blair, le 20 septembre 2001 à l'Elysée : « Dans l'hypothèse [d'une riposte américaine], il est évident [...] que ni l'Angleterre, ni la France ne pourraient être absentes ». 27. . Voir la déclaration finale du sommet, du 3 octobre 2001. 
28. . Voir H. Sjursen, K.E. Smith, « Justifying EU foreign policy : the logics underpinning EU enlargement », ARENA Papers, $\mathrm{n}^{\circ} 1,2001$.

29. . Voir T. Hopf (ed.), Understandings of Russian foreign policy, Philadelphia, Pennsylvania State University Press, 1999 ; ou J-P. Massias, « Russie : crise d'identité d'un Etat en recomposition ", in F. Charillon (ed.), Les politiques étrangères : ruptures et continuités, Paris, La Documentation Française, 2001.

30. . Voir la déclaration sur le Moyen-Orient du 29 octobre 2001. Les 15 ministres de l'Union Européenne se sont retrouvés les 5 et 6 novembre à Bruxelles avec leurs homologues du sud et de l'est de la Méditerranée, dans le cadre de la Conférence ministérielle EUROMED, afin de rappeler l'inéluctabilité du processus de Barcelone après les attentats du 11 septembre, et de discuter du processus de paix au MoyenOrient.

31. Sur l'après 11 septembre 2001 dans cette région du monde, voir le numéro spécial de Confluences Méditerranée, $n^{\circ}$ 40, hiver 2001-2002.

32. . Le président Bush a notamment relayé une demande d'Ariel Sharon à l'Union Européenne, de ne plus recevoir Yasser Arafat (demande heureusement rejetée par les Quinze). Les Etats-Unis ont ensuite opposé leur veto à un texte demandant l'établissement d'un « mécanisme de confiance » dans la région, parce qu'il ne plaçait pas la responsabilité exclusive des violences sur les Palestiniens. Veto que le ministre français des Affaires étrangères, Hubert Védrine, a jugé d'autant plus « incompréhensible » que les Etats-Unis avaient donné leur accord à cette proposition lors de la réunion du G8 à Vienne en juin dernier.

33. . La comparaison douteuse effectuée par le Premier ministre israélien, Ariel Sharon, entre Oussama ben Laden et Yasser Arafat, a été à juste titre rejetée par leurs chancelleries européennes, qui avaient pourtant mieux toléré celle effectuée par Vladimir Poutine entre le terrorisme d'Al Qaida et le « terrorisme tchétchène »... 34. . Voir F. Charillon, « La stratégie européenne dans le processus de paix du MoyenOrient : politique étrangère de proximité et diplomatie du créneau ", in M-F. Durand et A. de Vasconcelos, Une politique étrangère pour l'Europe Paris, Presses de Sciences Po, 1998, pp.195-225.

35. . Ibid.

36. . Voir M. Holland (ed.), Common Foreign and Security Policy : The record and reforms, London, Pinter, 1997.

37. . C'est l'Alliance du Nord qui s'est battu sur le terrain pour les Etats-Unis (à moins que ce ne soient les Etats-Unis qui aient bombardé pour l'Alliance du Nord...). Mais demain, en Irak peut-être, qui ira combattre Saddam Hussein, les Kurdes étant divisés, et les Chi'ites du Sud trop impuissants?

38. . Z. Laïdi, Géopolitique du sens, Paris, Desclée de Brower,1998.

\section{RÉSUMÉS}

Jusqu'où l'exceptionalisation au nom du péril national, invoqué par les autorités américaines, peut-elle s'étendre et durer ? Dans cet article, Frédéric Charillon souligne que cette question est 
loin d'être une question purement américaine et que les effets des politiques antiterroristes américaines touchent aussi l'Union Européenne. En premier lieu parce que les gouvernements européens ont voulu montrer leur solidarité et que, par la suite, la transnationalisation de la violence joue en faveur d'une transnationalisation des politiques de lutte contre le terrorisme et réenforce l'axe transatlantique. Frédéric Charillon insiste dans cet article sur les difficultés de l'Union Européenne à trouver une politique spécifique.

How far can exceptionalism - in the name of a national threat, as invoqued by the American authorities - spread and last ? Frederic Charillon highlights that this question is no exclusive American question as its effects on anti terrorist policies are felt within the European Union as well. This first occured because the European States were eager to show their solidarity and then because the transnationalisation of violence acts in favour of a transnationalisation of anti terrorist policies as well as it reinforces the trans-Atlantic axis. Frederic Charillon insists on the difficulties for the European Union to find a specific policy.

\section{INDEX}

Mots-clés : construction européenne, diplomatie, normes internationales, politique étrangère, Relations Internationales

Index géographique : Bruxelles, Union européenne

Thèmes : Union européenne (U.E.) 